\title{
Design of WSN with Relay Nodes Connected Directly with a LEO Nanosatellite
}

\author{
Adnane Addaim, Abdelhaq Kherras, and Zouhair Guennoun
}

\begin{abstract}
There have been a few studies in the literature about using a Low Earth Orbit (LEO) satellite as a component of wireless sensor network (WSN). The satellite, as small as a Nanosatellite with a lifetime of three months, requires employment of a very simple payload whereas all the complexity is brought back to the relay nodes (RNs) which are connected directly to the Nanosatellite using a random access protocol. The mainly constraint on the design of this type of $\mathrm{RN}$ is its energy consumption which determine the lifetime of the WSN network. In this article, in order to calculate the energy comsumption of each RN, we will establish a mathematical model which takes into account all parameters involved for determining the lifetime of a RN for direct Communication with a LEO Nanosatellite. This model is completted by using discret event simulator. The simulations show that we could reach the maximum number of RNs that can be served by the Nanosatellite by implementing an optimized method of energy-saving on these RNs.
\end{abstract}

Index Terms-LEO satellite, data aggregation, slotted ALOHA, WSN, satellite communication.

\section{INTRODUCTION}

Recent advances in the development of Wireless Sensor Networks (WSNs) [1] reveal a new architecture [2] for WSN network using direct communication between typical RNs and a satellite. In this paper, each RN plays the role of cluster head node which consists of aggregating received data from many sensor nodes into an aggregated data packet and then forwarding the aggregated data packet to the Nanosatellite.

The network architecture under consideration consists of SNs, RNs, a satellite and a Central Station (see Fig. 1). SNs located in specified predetermined sensing locations send the gathered physical information to a RN, which in turn forwards the data to the LEO Nanosatellite. Noted that the number of passes per day of the Nanosatellite, with altitude of $650 \mathrm{Km}$, over the same service area on the earth is equal to four [3]. A central station could gather data via the Nano- satellite from worldwide remote sites, where human presence is expensive and difficult to support (montains, deserts, oceans, dams, ...). Then the gathered data will be archived to a database and accessed by Internet. In this article, we will focus on the direct communication between the RNs and the Nanosatellite and especially on the energy consumption of a RN which is the key parameter to design such WSN.

Manuscript received February 12, 2014; revised April 28, 2014.

A. Addaim is with the Ecole Nationale des Sciences Appliquées (ENSA), Kenitra, B.P 241, Morocco (e-mail: addaim@gmail.com).

A. Kherras and Z. Guennoun are with the Mohammadia Engineering School, Rabat, B.P 765, Morocco (e-mail: kherras@emi.ac.ma, guennoun@emi.ac.ma).

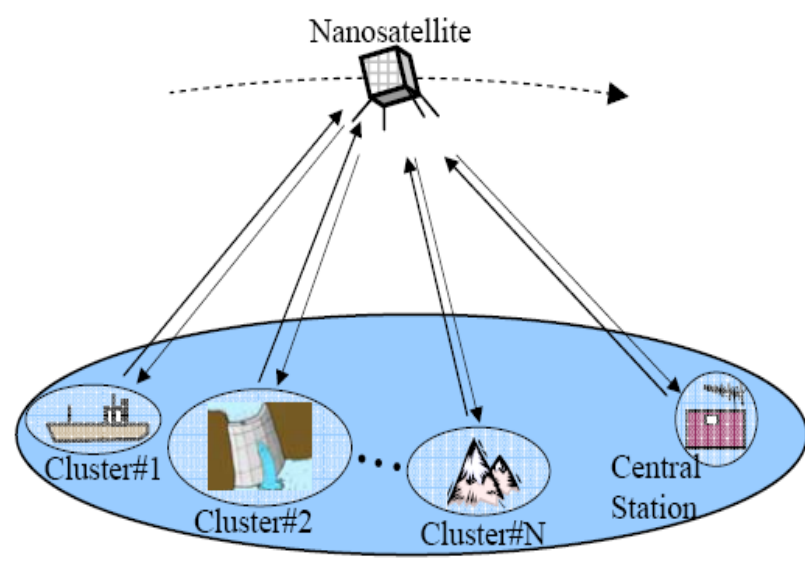

Fig. 1. WSN using store and forward nanosatellite.

\section{SYSTEM DESCRIPTION}

Consider a communication network comprising one LEO Store and forward Cubesat Nanosatellite and a finite number of RNs on the ground. The instantaneous coverage of the satellite depends on the minimum angle of elevation $E_{\min }$ under which any RN can be serviced by the Nanosatellite. Because of the spherical shape of the earth and natural obstacles, such as mountains, any location with an elevation angle less than $E_{\min }$ can not be easily seen from the Nanosatellite. In the satellite system model, we will assume a satellite in circular orbit at the altitude $h=650 \mathrm{~km}$ with a minimum elevation angle $E=10^{\circ}$.

The RNs use the Slotted ALOHA multiple access to send their packets to the Nanosatellite payload, which stores the correct packets in an on-board storage system, and delivers this to the destination Central Station in a later time. Between the storage and the retrieval of the packet, the Nanosatellite moves around its orbit and the earth rotates on its axis. These movements change the location of the Nanosatellite's footprint, and the Nanosatellite effectively carries the packet from RNs to the Central Station. The stored packets are to be formatted for download when the right telecommand is given from the Central Station.

With the assumption that RNs are distributed randomly in a given service area around the subsatellite line, the maxim- um time of the visibility $T_{s}$ for each $\mathrm{RN}$ is equal to 542 seconds whereas the global time $T_{s v}=2 T_{s}$ of the Nanosa- tellite visibility over its service coverage area is equal to $18 \min 4 \mathrm{~s}$ when $h=650 \mathrm{Km}$ and $E_{\min }=10^{\circ}$ [4]. Noted that, when the Nanosatellite altitude increases, the satellite visibi- lity time by RNs increases too. But, we choose the altitude of $650 \mathrm{~km}$ in order to keep the $\mathrm{RN}$ transmitted power acceptable and the launch cost of the Nanosatellite very low [5]. 


\section{SENSOR NODE DESIGN}

The Nanosatellite requires employment of a simple store-and-forward payload, due to the Cubesat dimensions of $10 \times 10 \times 10 \mathrm{~cm}^{3}$, whereas all the complexity is brought back to the RNs. The RNs are equipped with a Control Unit which uses the orbitography software to recognize the moments of the Nanosatellite passage. To make its calculations, the orbitography software needs three parameters to know, RN geographical position, UTC time and the NORAD-Keplerian Elements. The first two parameters are given by internal GPS receiver. The third parameter is given by the central station which regularly communicates ( 1 to 2 times per about fifteen days) the updated NORAD-Keplerian Elements of the Nanosatellite to all the RNs. The Fig. 2 illustrates the functional architecture of the RNs.

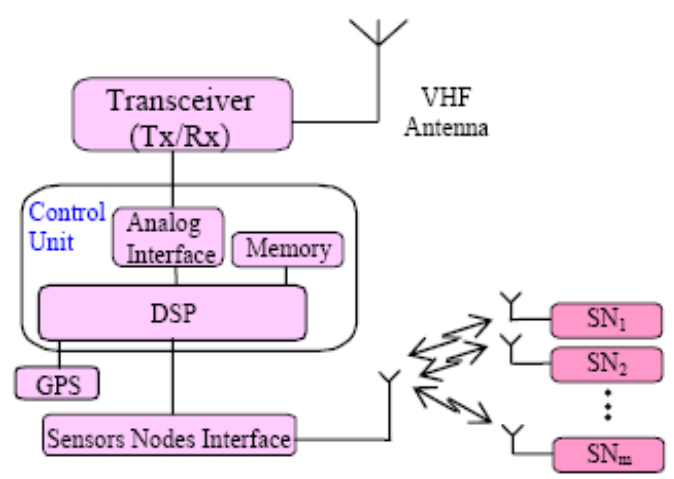

Fig. 2. Functional architecture of relay nodes.

The compact design of the RN facilitates its fixing aboard many types of payloads, including cargo containers, buoys, pipelines and boats. In fact, The RN proposed in this paper is based on the Nanosatellite electronic card payload [6] with the addition of a GPS board and a backup lithium battery instead of Nanosatellite electrical power subsystem. Its built-in software handles all functions involved in collecting, packaging, and transmitting the various data to the Nanosatellite from all SNs belong to its cluster. A satellite visibility calculation algorithm, developed in assembly language, had been loaded in the microcontroller memory to make the $\mathrm{RN}$ intelligent. This algorithm also allows the $\mathrm{RN}$ to operate in a very efficient battery power-save mode: the transmitter is keyed on only when the satellite is visible to the $\mathrm{RN}$. The implemented orbit model is a general model valid for low earth orbits with altitudes higher than $500 \mathrm{Km}$ to achieve a good prediction precision. The orbit calculation methodology used expresses the satellite coordinates in the $\mathrm{RN}$ relative topocentric coordinate system which easily yield the values of elevation and azimuth angles along with the slant range. This is achieved by means of satellite vector transformations through different coordinate systems [7].

To minimize the energy consumption of the RN battery and consequently to increase its exploitation duration, a method of energy-saving is implemented. It consists in managing the power ON and OFF of the following equipments; the GPS, the sensors Nodes Interface, the tranceiver, and the DSP. The sensors Nodes Interface and the GPS will be powered ON only at the times of measurements, the transceiver will be supplied only at the time of the communications sessions with the Nanosatellite and the DSP will function in standby mode. The operation of the RN is thus schematized by the algorithm of the Fig. 3.

Outside the moments of sensing and measurement from SNs and GPS, the DSP goes to the standby mode when the RN is not in visibility with the Nanosatellite. In this mode, the CPU, the clock and all the internal modules of the DSP are inactive in order to reduce the energy consumption to a very low level. The DSP awakes at the end of each 10 seconds to check if the moment is arrived to take sensing and measurements from SNs and GPS or to communicate with the Nanosatellite otherwise it returns to the standby mode.

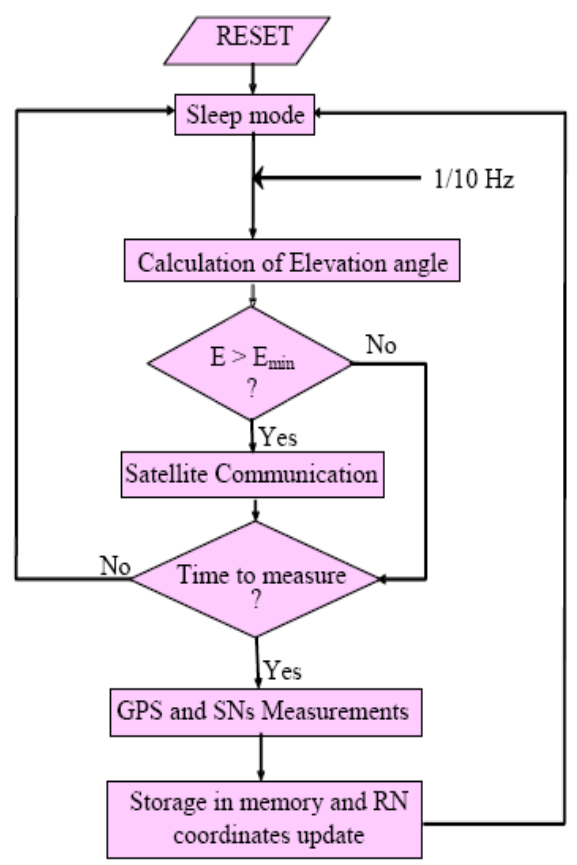

Fig. 3. Relay nodes flowchart.

Outside the Nanosatellite visibility, the $\mathrm{RN}$ receives packets from all SNs in its cluster through SNs Interface. The RN implements aggregation techniques in such way the volume of the received data, from all SNs in its cluster, is equal to the volume to be transmitted successfully by the RN to the Nanosatellite during its visibility time $T_{s}$.

\section{RADIO LINK}

At VHF frequencies the atmosphere and ionosphere have little effect on the propagating radio wave [8]. Also, antennas, receivers and transmitters for both the ground and the space segment are readily available and inexpensive.

The link budget, shown in Table I, demonstrates how an acceptable service can theoretically be obtained, using GMSK modulation [6], between a Nanosatellite with simple omnidirectional antenna, and a RN with quarter wavelength antenna. We use only one frequency for both uplink and downlink in order to keep the system overall cost very low. If one looks at the cubesat projects they generally use about one watt of output power of the Nanosatellite while limiting the data rate to 1200 baud. The Doppler shift is about $\pm 3 \mathrm{KHz}$ maximum, which can be ignored for the Nanosatellite parameters with altitude of $650 \mathrm{Km}$ and operating frequency of $145.825 \mathrm{MHz}$. 
TABLE I: BUDGET LINK Sensor Node Parameters

\begin{tabular}{lc}
\hline \multicolumn{2}{c}{ Sensor Node Parameters } \\
\hline Antenna gain & $0 \mathrm{dBi}$ \\
Transmitted power & $1 \mathrm{~W}$ \\
Antenna Feed Loss & $0.5 \mathrm{~dB}$ \\
$\mathrm{~T}_{\text {syst }}$ & $2000 \mathrm{k}$ \\
\hline \multicolumn{2}{c}{ Channel Parameters } \\
\hline Free Space Loss & $-141 \mathrm{~dB}$ \\
Polarization Losses & $3 \mathrm{~dB}$ \\
Additional Losses & $0.5 \mathrm{~dB}$ \\
\hline \multicolumn{2}{c}{ Satellite Parameters } \\
\hline Antenna gain & $0 \mathrm{dBi}$ \\
Transmitted power & $1 \mathrm{~W}$ \\
Antenna Feed Loss & $0.5 \mathrm{~dB}$ \\
$\mathrm{~T}_{\text {syst }}$ Temprature & $5000{ }^{\circ} \mathrm{k}$ \\
\hline \multicolumn{2}{c}{ Operational Parameters } \\
\hline Baud Rate & $1200 \mathrm{baud}$ \\
$\mathrm{E}_{\mathrm{b}} / \mathrm{N}_{0}$ required & $13.6 \mathrm{~dB}$ \\
\hline \multicolumn{2}{c}{ Link Margin for Uplink } \\
\hline $\mathrm{E}_{\mathrm{b}} / \mathrm{N}_{0}$ estimated & $25.9 \mathrm{~dB}$ \\
Margin & $12.3 \mathrm{~dB}$ \\
\hline \multicolumn{2}{c}{ Link Margin for Downlink } \\
\hline $\mathrm{E}_{\mathrm{b}} / \mathrm{N}_{0}$ estimated & $22.9 \mathrm{~dB}$ \\
Margin & $9.3 \mathrm{~dB}$ \\
\hline
\end{tabular}

To reduce the transmit power under 1 Watt in order to increase the operation life of the batteries, we could increase the $E_{\min }$, but the Nanosatellite time visibility decreses and hence the number of possible deployed RNs decreses too. So, the use of LEO Nanosatellites constellation will be the solution in order to maintain the number of possible deployed RNs high although we will icrease the $E_{\text {min }}$. Noted that the use of $1 \mathrm{~W}$ of transmitted power is equivalent to current consumption of $1.5 \mathrm{~A}$ by the $\mathrm{RN}$.

\section{RELAY NODES NANOSATELLITE COMMUNICATION}

The share of the Nanosatellite channel by all the RNs on the ground is done according to the Slotted ALOHA protocol. All the RNs transmit their packets at common clock instances without caring about the other RNs. The impulsion per second generated by the GPS is used to synchronise the transmission of the packets. When a collision occurs the packet is retransmitted after a random interval time (see Fig. 4).

Once the satellite comes into range, the RN starts a communication session by transmitting a packet of duration $T_{\text {inf }}$ using a Send-and-Wait ARQ protocol. The packet format follows the AX.25 packet format [9]. In the case of the packet successful reception, the Nanosatellite sends an acknowledgment packet of duration $T_{\text {ack }}$. For LEO satellite systems, ALOHA protocol should have promise, even though its basic idea may require some modifications [10]. The implementation of the Slotted ALOHA in our design has the advantage to use only one frequency band for both the uplink and the downlink. Taking advantage of the half-duplex communications, during one slot the RN could transmit and receive the Nanosatellite reply with the same frequency band (see Fig. 4). This procedure avoids the loss of the acknowledgment packet due to collisions with the information packets sent by other RNs because they are all synchronised (see Fig. 4).

The duration of a slot $\tau$ is given by the following relation.

$$
\tau=T_{\text {up }}+T_{\text {inf }}+T_{\text {tr }}+T_{\text {ack }}+T_{\text {down }}
$$

with $T_{\text {up }}$ and $T_{\text {down }}$ are the propagation time for uplink and downlink respectively. They are obtained by dividing the satellite range by the speed of light: the maximum is about 6.8 $\mathrm{ms}$ at $10^{\circ}$ elevation, which is negligible compared to the packet duration $T_{\mathrm{inf}}=1.66 \mathrm{sec}$, which is obtained by dividing the packet length $L=253$ bytes by the transmission rate $R=1200 \mathrm{bps}$. The duration of the acknowledgment packet is $T_{\text {ack }}=0.126 \mathrm{sec}$, by using an acknowledgment packet length equals to 19 bytes. According to (1), the duration of a slot is $\tau$ $=2 \mathrm{sec}$, with the assumption that the processing time on board the Nanosatellite is $T_{\mathrm{tr}}=200 \mathrm{~ms}$. For the sake of simplicity in calculations, Zero-guard time is assumed here. If the acknowledgement packet is not received, a retransmission of the same packet is carried out after a random time $T_{\mathrm{r}}$.

In the case of Slotted ALOHA, we have $T_{\mathrm{r}}=\delta \tau$, with $\delta$ is the number of timeslots to wait for retransmission. $\delta$ is an integer number chosen uniformly in the interval $\left[0, \delta_{\max }\right]$.

The number of the timeslots $\beta$ available during the period of the satellite visibility by each $\mathrm{RN}$ is given by the following relation:

$$
\beta=T_{s} / \tau
$$

In our case, we find that $\beta=271$ timeslots. So, the total number of timeslots available during the satellite pass over the service area is equal to $2 \beta=542$ timeslots, taking into account the time shift between the two extreme points of the satellite service area which equals to $T_{s}$.

In Slotted ALOHA random access protocol, the throughput $\mathrm{S}$ is defined as the expected number of successfully packets in each time slot and the traffic load $\mathrm{G}$ is defined as the number of newly generated and retransmitted packets in each time slot. Since all RNs are identical, we could define the normalised $G$ and the normalised $S$ as

$$
G=N_{t} / 2 \beta \quad \text { and } S=N_{s u c} / 2 \beta
$$

where, $N_{\mathrm{t}}$ is the total number of transmitted packets (new and retransmitted packets) during the time of visibility of the satellite, and $N_{\text {suc }}$ is the number of successfully transmitted packets during the time of visibility of the satellite. On the one hand, in the real experimental environment, the total number $N_{t}$ is determined by the configuration of the same $\delta_{\max }$ in all RNs. But on the other hand, the $N_{\text {suc }}$ is very difficult to figure out by an analytical method as a function of the $\delta_{\max }$ [11]. So, several simulations using NS2 simulator [12] have been made to find out the maximum number of RNs that can be deployed over a given geographical service area of the Nanosatellite. Using the Slotted ALOHA protocol as described before, the optimization parameter, to reach the maximum throughput and the minimum energy consumption, is the maximum number of timeslots $\delta_{\max }$ to wait for retransmission. The result of the simulations is given by Fig. 5 which shows the number of packets received succeefully on board the Nanosatellite versus values of $\delta_{\max }$ for four different numbers of RNs.

For small values of $\delta_{\max }$, the traffic is heavy which increases the number of retransmissions and hence increases the energy consumption. In this case, the amount of the successfully 
transmitted packets is small and doesn't reach the maximum throughput. However, For bigger values of $\delta_{\max }$, the traffic is low and the number of retransmissions decreases but the drawback here is a low value of the successfully transmitted packets because many packets don't have a chance to be retransmitted due to the limited visibility time $T_{s}$.

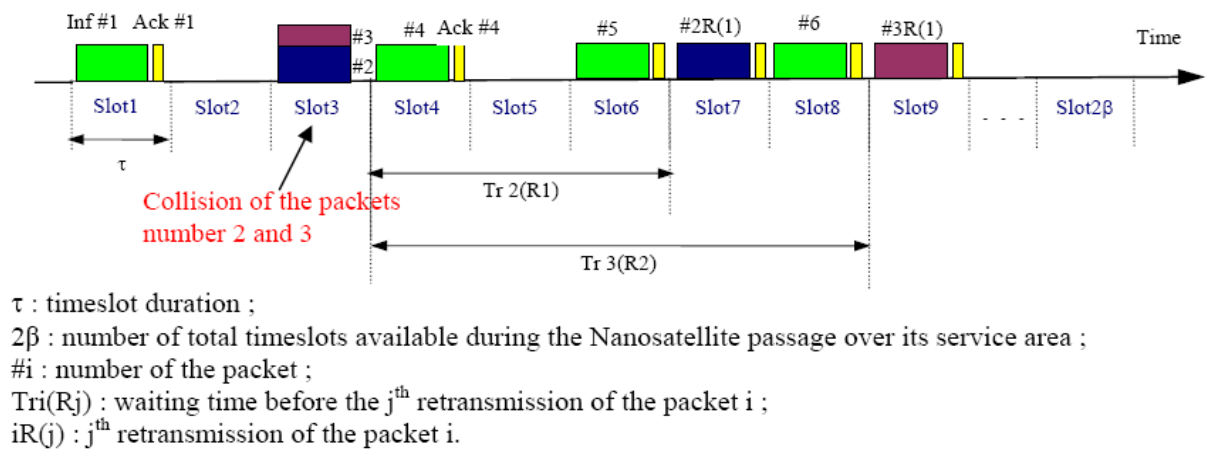

Fig. 4. RelayNodes-Satellite communication session.

The maximum number of successfully received packets is reached for an optimum $\delta_{\max }$ which depends on the number of RNs on the same service area of the Nanosatellite. For the required traffic, the simulations in Fig. 5 show a stable network with long interval of $\delta_{\max }$ where the number of RNs is less than 175. If the value of $\delta_{\max }$ exceeds 150 for $N_{s}=175$, the throughput of the system doesn't reach its maximum. Using 200 RNs over the same service area of the Nanosatellite makes the network unstable for any value of $\delta_{\max }$.

The condition of the stability [13] states that the departure rate $\lambda$ (new packets rate) is equal to the arrival rate $S$ (Throughput).

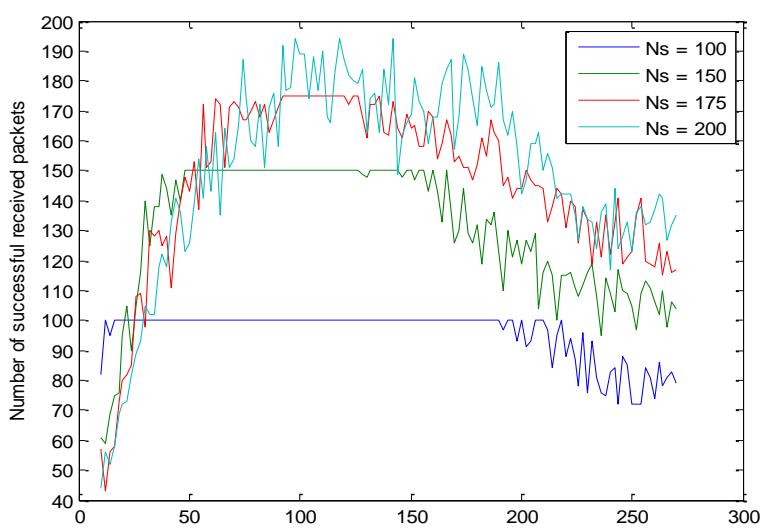

The maximum number of timeslot $\delta$ max to wait for retransmission

Fig. 5. Number of packets received successfully on board the Nanosatellite as a function of $\delta_{\max }$.

In the model presented in this article, it is assumed that each $\mathrm{RN}$ transmits at the maximum one packet during the visibility period of the satellite. So, with $\lambda<S_{\max }$, we could define $\lambda$ by the expression below:

$$
\lambda=N_{s} / 2 \beta
$$

In our case, with $\beta=271$, the network will be unstable for the required traffic when the number of terminal exceeds 200 RNs, as shown in Fig. 5. In the stable case where $\lambda<S_{\max }$ with $\beta=271$ timeslots, the maximum number of RNs that could be deployed at the same service area of the Nanosatellite is around 175 so that the system could possess a stable equilibrium point (see Fig. 5).

In the stable case where $\lambda<S_{\max }$ with $\beta=271$ timeslots, the maximum number of RNs that could be deployed theorically at the same service area of the Nanosatellite is 195 , but in this case, the system doesn't possess any stable equilibrium point. This result is in good correlation with the simulations represented by the Fig. 5 which shows that the network is always unstable for the required traffic when the number of RNs is equal to 200 .

The average of the probability of packet success $P_{\text {suc }}$ during the visibility period of the satellite is given by:

$$
P_{\text {suc }}=\frac{\lambda}{G}
$$

with $(\mathrm{G}, \lambda)$ stable equilibrium point.

From (3), (4) and (5), we have:

$$
P_{s u c}=\frac{1}{N_{t p}}
$$

with $N_{\mathrm{tp}}$ is a real number which represents the average number of transmissions of the same packet (new and retransmitted packets) per each RN. This number is obtained by dividing $N_{\mathrm{t}}$ by $N_{\mathrm{s}}$. In general, when $N_{\text {tp }}$ is equal to one (as in TDMA) means that there is no retransmission of the new packet and hence the probability of success is always equal to one which is intuitively reasonable. But in Slotted ALOHA multiple access, this number is always greater than one.

In the standard Slotted Aloha random access protocol, the maximum throughput 0.36 is reached for $G=1$. In this case, the probability of success and the number $N_{t p}$ are equal respectively to 0.36 and 2.77 [10].

We mentioned here that in practice, the number $N_{t p}$ is related to the energy consumption. In fact, while the RN transmitter is requested $N_{t p}$ times to transmit the same packet means that the battery supplies $N_{t p}$ times the transmitter to transmit this same packet.

As shown in the Fig. 6, the number $N_{\text {tp }}$ decreases when the value of $\delta_{\max }$ increases, but for high values of $\delta_{\max }$, the system will be unstable (see Fig. 5). So, it is preferable to program the RNs with optimized value of $\delta_{\max }$ which is the higher value of $\delta_{\text {max }}$ that we could use otherwise the system will be unstable. For example, when the number of RNs is about 175 , we could use a value of $\delta_{\max }=120$ which corresponds to $N_{\mathrm{tp}}=2.4$. To increase the lifetime of the $\mathrm{RN}$ by reducing the energy consumption, we could use only $100 \mathrm{RNs}$ by programming the value of $\delta_{\max }$ to be equal to 190 which corresponds to 
$N_{\mathrm{tp}}=1.23$.

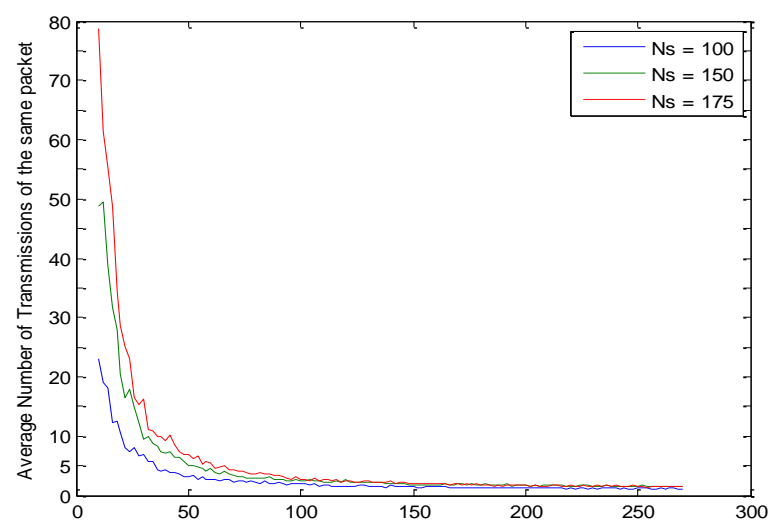

The maximum number of timeslot $\delta$ max to wait for retransmission Fig. 6. Average number of transmission of the same packet per each RN as a function of $\delta_{\max }$.

\section{RELAY NODE LIFE TIME}

The lifetime of the Nanosatellite is about three months [6]. In this section, we will calculate the energy consumption of each $\mathrm{RN}$ to be deployed on the ground in operation mode. We have to accommodate the deployment duration of each RN to be about three months according to a given scenario. And therefore, the capacity of the RN battery $C$ (expressed in A.H) must be determined to perform this scenario. To calculate the $\mathrm{RN}$ deployment duration in terms of number of days, we will firstly calculate the energy consumption of the RN per day for a given scenario which depends on the amount of the sensing informations from SNs and GPS.

We will use the following scenario, the deployed RN will operate in cyclic standby mode (1 second ON, 10 seconds OFF) and for each 24 minutes, it will take measurements from GPS and all SNs in its cluster during 18 seconds (sensing mode). Also, the transmitter-receiver will be turned ON only during the satellite communication session (Transmission mode and Reception mode).

During its deployment, the $\mathrm{RN}$ will pass in a repetitive way by four different operating modes; the standby mode, the active mode, the sensing mode, and the satellite communication session mode (transmission mode and reception mode). The current consumption from the battery depends on the operating mode of the RN and is determined by the sum of the currents of its various parts (control unit, Sensors Nodes Interface, GPS and Tx/Rx) which are active during this operating mode. The following Table II summarizes the energy consumption according to each operating mode of the RN.

\begin{tabular}{|l|c|c|c|c|c|}
\multicolumn{1}{c|}{} & \multicolumn{1}{c|}{ TABLE II: CURRUNT CONSUMPTION OF THE RN } \\
\cline { 2 - 6 } \multicolumn{1}{c|}{} & $\begin{array}{c}\text { Control } \\
\text { Unit }\end{array}$ & $\begin{array}{c}\text { SNs } \\
\text { Interface }\end{array}$ & GPS & Tx/Rx & $\begin{array}{c}\text { Current } \\
\text { consumption }\end{array}$ \\
\hline $\begin{array}{l}\text { Standby } \\
\text { Mode }\end{array}$ & off & off & off & off & negligible \\
\hline Active Mode & on & off & off & off & $10 \mathrm{~mA}$ \\
\hline Sensing mode & on & on & on & off & $230 \mathrm{~mA}$ \\
\hline $\begin{array}{l}\text { Transmission } \\
\text { mode }\end{array}$ & on & off & off & on & $\begin{array}{c}1.5 \mathrm{~A} \\
(1 \mathrm{Watt} \mathrm{RF})\end{array}$ \\
\hline $\begin{array}{l}\text { Reception } \\
\text { mode }\end{array}$ & on & off & off & on & $140 \mathrm{~mA}$ \\
\hline
\end{tabular}

\section{A. Energy Consumption per Day of SNs Interface and GPS}

The sensors nodes interface and GPS are turned ON only at the times of the Sensing mode and its energy consumption per day $\mathrm{E}_{\mathrm{S}}$ can be given by the following formula:

$$
E_{s}=\left(N_{m} \cdot t_{m} \cdot I_{s}\right) / 3600
$$

with $N_{\mathrm{m}}$ : the number of sensing per day

$t_{\mathrm{m}}:$ the duration of the measurements (in seconds)

$I_{\mathrm{S}}$ : the current consumption of sensors and GPS (in A).

The length of the information packet in AX.25 protocol depends on the length of the information field $N_{1}$ by $L=$ $\left(27+N_{1}\right)$ bytes with the maximum value of $N_{1}$ is equal to 256 and the 27 bytes is reserved to the overhead, which contains the addresses of the RN and the Nanosatellite, and other fields related to the control of the communication. We will use the length $N_{1}$ of 225 bytes so that the percentage of the overhead is around $10 \%$ of the whole packet which is desirable for the efficiency of the communication protocol. As mentioned before each RN transmits at the maximum one packet during the visibility period of the satellite and the number of the LEO satellite passes over the same service area per day is equal to four. So, the amount of the sensing data during a day is limited to 900 bytes. To improve it, we need to use an efficient multiple access protocol like CSMA/CA.

The length $N_{1}$ is related to the number of sensing per day $N_{m}$ by:

$$
N_{p} . N_{1}=N_{m} \cdot N_{b m}
$$

with $N_{\text {bm }}$ : number of Bytes per measurements

$N_{\mathrm{p}}$ : number of Nanosatellite passes per day.

We assume that the RN will perform over one day 60 measurements from sensors nodes via any wireless Adhoc protocol (like Zigbee) and from GPS. The sensing mode lasts 18 seconds with a current consumption of $230 \mathrm{~mA}$. The daily consumption of the Sensors Nodes Interface and the GPS will be thus equal to $0.11 \mathrm{AH}$.

\section{B. Energy Consumption per Day of Control Unit}

The control unit functions only 1 second over 10 using the standby mode and consumes $10 \mathrm{~mA}$. During one day, it will consume 0.024 A.H which is calculated according to the following formula:

$$
E_{c u}=24 . \text { Cycle }_{s t b} . I_{c u}
$$

with Cycle $_{\text {stb }}$ : the stand-by cycle (is equal to $1 / 10$ )

$I_{\mathrm{cu}}$ : the current consumption of the $\mathrm{CU}$ (in A).

\section{RN Energy Consumption during Satellite Communication Session}

In this subsection, we evaluate the energy consumption of the $\mathrm{RN}$ during the $\mathrm{RN}$-satellite communication session which is equal to the energy for sending one newly generated data packet until its successful reception by the Nanosatellite including the retransmitted packets after collisions. It is obviously that the transmitter is turned $\mathrm{ON}$ only at the moment of the sending of the data packet during the satellite visibility and the receiver is turned $\mathrm{ON}$ after each 
transmission to receive the acknowledgment packet. In the case of successful reception of the newly generated data packet by the Nanosatellite, the $\mathrm{RN}$ receives the acknowledgment packet after the beginning of the following slot (see Fig. 4). After this first successful attempt, the RN clears the data packet and the energy consumed in this case is $E_{1}=T_{i n f} . I_{t r}+\left(\tau-T_{i n f}\right) . I_{r c}$ with $I_{t r}=1.5 \mathrm{~A}$ is the current consumed during the RF transmission and $I_{r c}=140 \mathrm{~mA}$ is the current consumed during the RF reception. So, $E_{1}$ is equal to 0.7 $\mathrm{mA}$.H. If the acknowledgment packet is not received, the RN considers the packet lost and starts the process of reattempting transmission after a random interval time (see Fig. 4).

As noted before, to minimize the energy consumption, the lost packet is retransmitted with high optimized value of $\delta_{\max }$ that yields to a low value of average number of transmission of the same packet $N_{t p}$. The average energy consumption of the $\mathrm{RN}$ during the $\mathrm{RN}$-satellite communication session $E_{\mathrm{SC}}$ can be expressed as:

$$
E_{S C}=\sum_{k=1}^{\infty}\left(E_{1}+k E_{w}\right)\left(1-P_{\text {suc }}\right)^{k} P_{\text {suc }}
$$

where $E_{\mathrm{w}}=E_{1}+\delta_{\mathrm{moy}} \cdot \tau \cdot I_{\mathrm{rc}}$ is the average energy consumed between the end of the first transmitted packet and the end of the first retransmitted packet or between the ends of any two successive retransmitted packets. And $\left(1-P_{\text {suc }}\right)^{k} P_{\text {suc }}$ is the probability that a packet being received in the $(k+1)^{\text {th }}$ attempt of transmissions after $k$ times of retransmissions failures, And $E_{1}+k E_{\mathrm{w}}$ is the average energy consumed between the generation of the packet and his full acceptance after $(1+k)^{\text {th }}$ attempt.

By developing the (10) and using the properties of the geometric series and the derivative functions, we will obtain:

$$
\begin{aligned}
& E_{\text {SC }}=E_{1} P_{\text {suc }} \sum_{k=1}^{\infty}\left(1-P_{\text {suc }}\right)^{k}+E_{w} P_{\text {suc }}\left(1-P_{\text {suc }}\right)\left(\sum_{k=1}^{\infty}\left(1-P_{\text {suc }}\right)^{k}\right) \\
& =E_{1}+E_{w}\left(1-P_{\text {suc }}\right) \frac{1}{P_{\text {suc }}}
\end{aligned}
$$

By replacing $P_{\text {suc }}$ and $E_{\mathrm{w}}$ by their expressions, we will find:

$$
E_{S C}=E_{1} \cdot N_{t p}+\frac{\delta_{\max }}{2} \cdot \tau \cdot I_{r c} \cdot\left(N_{t p}-1\right)
$$

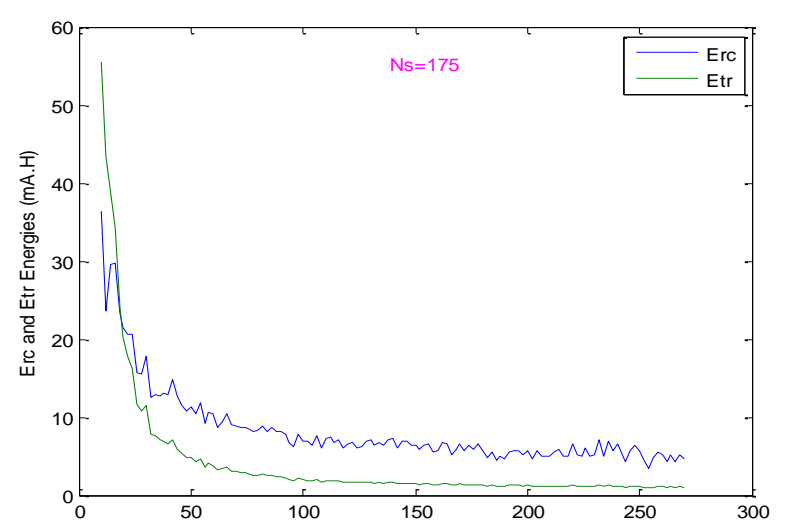

The maximum number of timeslot $\delta$ max to wait for retransmission Fig. 7. $E_{r c}$ and $E_{t r}$ energies as a function of $\delta_{\max }$ for $N_{s}=175$.
The first term $E_{\mathrm{tr}}=E_{1} \cdot N_{\mathrm{tp}}$ of (12) represents the average consumed energy due to the transmission of the same paquet $N_{\mathrm{tp}}$ times, whereas the second term, $E_{\mathrm{rc}}=\delta_{\text {moy }} \cdot \tau \cdot I_{\mathrm{rc}} \cdot\left(N_{\mathrm{tp}}-1\right)$, represents the average consumed energy due to the random waiting time before retransmissions. The Fig. 7 shows that the consumed energy $E_{\mathrm{rc}}$ is bigger than the consumed enegy $E_{\mathrm{tr}}$ for $N_{s}=175$ RNs. It represents more than $80 \%$ of the whole energy consumption $E_{\mathrm{SC}}$ for $N_{\mathrm{s}}=175$ and $\delta_{\max }>112$.

To optimize the energy consumption, the receiver will be turned OFF during the random waiting time $T_{\mathrm{r}}$, and therefore the energy consumption $E_{\mathrm{SC}}$ will be reduced to the consumed energy $E_{t r}$ (see (13)) which doesn't depend explicitly on the number of RNs and the maximum number of waiting timeslot $\delta_{\max }$ but it depends implicitly on them from $N_{t p}$. Using this technique, the expression of the optimized RN energy consumption $E_{S C}^{\prime}$ will be deduced from (12) as follow:

$$
E_{S C}^{\prime}=E_{1} \cdot N_{t p}=E_{1} / P_{s u c}
$$

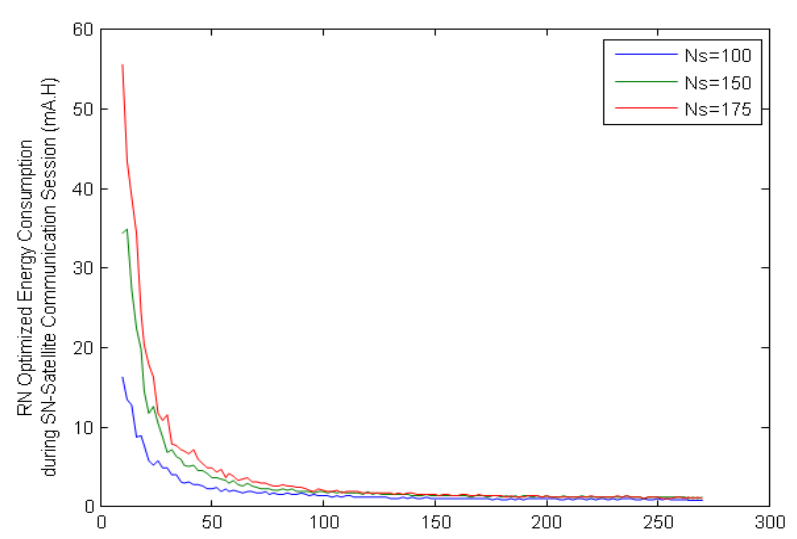

The maximum number of timeslot $\delta$ max to wait for retransmission

Fig. 8. RN optimized energy consumption during SN-satellite communication as a function of $\delta_{\max }$.

The Fig. 8 shows the RN optimized energy consumption as a function of $\delta_{\max }$. As mentioned before, to get the minimum of number of $N_{t p}$ during the RN-Satellite communication session, it is preferable to program the RNs with optimized value of $\delta_{\max }$ which is the higher value of $\delta_{\max }$ that we could use otherwise the system will be unstable. For example, when the number of RNs is about 150, the optimized value of $\delta_{\max }$ is equal to 120. Using Fig. 8, this case corresponds to the optimized energy consumption of $1.69 \mathrm{~mA} . \mathrm{H}$ during the RN-Satellite communication session. When we use only 100 SNs, the optimized value of $\delta_{\max }$ is 190 , which corresponds to the optimized energy consumption of $0.87 \mathrm{~mA} . \mathrm{H}$.

\section{The Life Time of Each RN}

The total energy consumption of the RN per day $E_{\mathrm{T}}$ is equal to the sum of the energies consumed by different operation modes:

$$
E_{T}=E_{u c}+E_{S}+4 \cdot E_{S C}^{\prime}
$$

The consumed energy $E_{\mathrm{s}}$ represents $79 \%$ of the total energy consumption $E_{\mathrm{T}}$. So, the lifetime of the deployed RN on the ground depends mainly on the consumed energy $E_{\mathrm{S}}$ due to the sensing from SNs and GPS. This lifetime is expressed in a 
number of deployment days $N_{\mathrm{D}}$ and is given by the following formula:

$$
N_{D}=\frac{C}{E_{T}}
$$

with $C$ : capacity of the battery (in $\mathrm{AH}$ )

$E_{\mathrm{T}}$ : total energy consumption of the RN per day (in $\mathrm{AH}$ ).

For the proposed scheme with $N_{\mathrm{s}}=175$, the RN consumes $0.14 \mathrm{AH}$ per day according to (14). A battery with capacity of $12 \mathrm{AH}$ allows the RN to function in a continuous way for 86 days. The same battery capacity of $12 \mathrm{AH}$ and by using only $100 \mathrm{SNs}$, the lifetime of the $\mathrm{RN}$ remains almost the same about 87 days. We could explain this result by the fact that the total energy consumption of the RN per day $E_{T}$ depends little on the consumed energy $E_{\mathrm{SC}}$ which depends on the number of RNs over the same service area of the Nanosatellite. The evolution of the battery level nevertheless is supervised by the central station from the data received from the RN and the decision to repatriate the dead $\mathrm{RN}$ can be made at the adequate time.

\section{CONCLUSION}

The LEO satellite will be the main component of the future WSN network because it is not affected by the natural disasters and could keep the WSN network at the extreme low end of the satellite communications cost spectrum by using a low-cost Nanosatellite. In this paper, we proposed a method to save the energy consumption for deployed relay nodes. This method is validated by several simulations which take into account all parameters involved for determining the energy consumption of a relay node for direct communication with LEO Nanosatellite. Using this method, we could reach the maximum throughput of the Slotted ALOHA without increasing the energy consumption of the sensor nodes. As a future work, we will focus on developing a multiple access protocol with high probability of success, rather than the Slotted Aloha protocol proposed in this paper, in order to increase the amount of collected sensing data to be transmitted to the Nanosatellite by each relay node.

\section{REFERENCES}

[1] I. Akyildiz, W. Su, Y. Sankarasubramaniam, and E. Cayirci, "A survey on sensor networks," IEEE Communications Magazine, vol. 40, no. 8, pp. 102-114, 2002.

[2] N. Celandroni, E. Ferro, A. Gotta, G. Oligeri, C. Roseti, and M. Luglio et al., "A survey of architectures and scenarios in satellite-based WSN," International Journal of Satellite Communications and Networking, vol. 31, pp. 1-38, 2012.

[3] B. Zantou, A. Kherras, and A. Addaim, "Performance evaluation of a single microsatellite data collection system using small ground terminals," Journal of Aerospace Computing, Information and Communications, vol. 3, pp. 63-72, 2006.

[4] A. Addaim, A. Kherras, and B. Zantou, "Design and analysis of store-and-forward data collection network using low-cost small satellite and intelligent terminals," Journal of Aerospace Computing, Information and Communications, vol. 5, no. 2, pp. 35-46, 2008.

[5] R. J. Twiggs and S. Nakatsuka et al., "A cubesat: A new generation of picosatellite for education and industry low-cost space experimentation," in Proc. 14th Annual AIAA/USU Conference on Small Satellites, Logan, Utah, August 2000.
[6] A. Addaim, A. Kherras, and B. Zantou, "DSP implementation of integrated store-and-forward APRS payload and OBDH subsystems for low-cost small satellite," Journal of Aerospace Science and Technology, vol. 12, pp. 308-317, 2008.

[7] W. L. Pritchard, H. A. Suyderhoud, and R. A. Nelson, Satellite Communication Systems Engineering, 2nd ed., Prentice Hall, ch. 2-3, 1993.

[8] J. Paffet, T. G. Jeans, and J. Ward, "VHF-Band interfrence avoidance for next-generation small satellites," in Proc. 12 th AIAA/USU Conference on Small Satellites, SSC98-II-5, 1998.

[9] TAPR, AX.25 Link Access Protocol for Amateur Packet Radio, TAPR, 2.2 edn., 1997.

[10] A. Jamalipour, Low Earth Orbital Satellites for Personal Communication Networks, Norwood, MA: Arthech House, 1998.

[11] F. A. Tobagi, M. Gerla, R. W. Peebles, and E. G. Manning, "Modeling and measurement techniques in packet communication networks," Proceedings of IEEE, vol. 66, no. 11, 1978.

[12] Network simulator NS-2. [Online]. Available: http://www.isi.edu/nsnam/ns.

[13] L. M. James, "Some new approaches to random-access communications," IEEE Press, Multiple Access Communications, pp. 354-368, 1992.

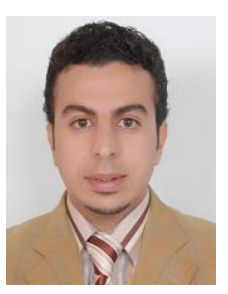

A. Addaim was born in Casablanca, Morocco, in 1975. He received his master diploma in 2001 and his $\mathrm{Ph} . \mathrm{D}$. degree in 2008, both in satellite communication from Mohammadia School of Engineers (EMI), Morocco. From 2002 to 2003, he was employed at Siemens.AG, as a transmission engineer, responsible of the implementation of the supervision network of the Meditelecom GSM network, in Morocco. From 2004 to 2009, he was a teaching assistant at EMI and research engineer at Centre for Space Research and Studies (CRES, EMI) working on research projects dealing with the design of university microsatellite. He is recipient of the first prize of the 2007-2009 best PhD thesis in the field of Sciences and technologies at Mohammed V University. From Mars to August 2010, he was with post-doctoral scholarship at the Satellite Communication and Networking Laboratory (SCNL) at the Faculty of Engineering, University of Genoa. From September 2010, he has been an assistant professor at the ENSA kenitra, Morocco. His current research interests include communication networks and satellite communication systems.

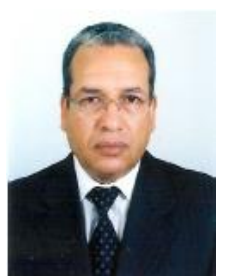

A. Kherras was born in Marrakech, Morocco, in1949. He received the master degree in EEA (Electronic Electrotechnic and Automatic) from Paul Sabatier University, Toulouse, the doctorate thesis in USTL, Montepellier University, in 1980, and the Ph.D degree from the Liege University, Belgium, in 1990. Since 1982, he has been a professor of Electronics in Electrical Engineering Department of EMI. He is currently the director of Centre for Space Research and Studies (CRES), and National Coordinator of University Network of Space Techniques and Sciences (RUSTE). His current research interests include space technology and satellite communication systems.

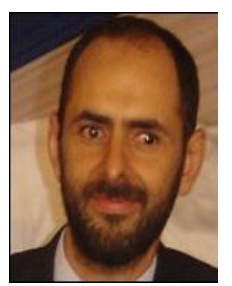

Z. Guennoun received his engineering degree in electronics and telecommunications from the Electronics and Electrical Montefiore Institute, ULG Liege, Belgium in 1987; his M.Sc. degree in communication systems from the EMI School of Engineering, Rabat, Morocco in 1993; and his PhD degree from the same school in 1996. He visited the Centre for Communication Research (CCR) in Bristol University, UK, during the period of 1990-1994 to prepare a split PhD. During 1988-1996 he worked as an assistant lecturer in the EMI School of Engineering, and from 1996 he is working in the same school as a professor lecturer. His fields of interest are digital signal processing, error control coding, speech and image processing. Currently he is in charge of the Research Laboratory in Electronics and Telecommunications (LEC) at EMI. He is an IEEE senior member (member since 1990); and member of the Moroccan IEEE section executive committee. 